\title{
Gibt es eine Insulinallergie?
}

\section{Hier steht eine Anzeige.}

Frage: Eine Diabetikerin (65) gibt eine Allergie auf sämtliche Analog- und Humaninsuline an; sie reagiere mit Hautausschlag und Spastik. Sind ähnliche Fälle bekannt? Gibt es eine Alternative?

Prof. Dr. Stephan Martin, Düsseldorf: Reine Insulinallergien sind sehr selten, aber diese gibt es. Häufiger sind jedoch Allergien gegen Zusatzstoffe in den Insulinpräparationen. Bei verschiedenen Diabeteszentren werden standardisierte Allergietestungen durchgeführt. Bei nachgewiesener Allergie gibt es Desensibilisierungsprogramme. Je nach Diabetes-Typ (Typ 1) gibt es sonst leider keine therapeutischen Alternativen. Bei Typ 2 Diabetes sollte geprüft werden, ob die OAD-Therapie kombiniert mit nicht-pharmakologischen Maßnahmen optimiert werden könnte.

\section{Was schützt vor Insektenstichen?}

Frage: Bei mir in der Praxis kommen häufiger Fragen zu Mückenschutz, bei denen ich mir nicht sicher bin. Offenbar wird vielen Patienten gesagt, dass Autan ${ }^{\varpi}$ und ähnliche Standardrepellents nicht ausreichend wirksam seien. Ihnen wird empfohlen, DEET-haltige Produkte zu verwenden. Soweit ich weiß, fallen diese aber durch eine hohe allergene Potenz, Hautreizungen und neurologische $\mathrm{Ne}$ benwirkungen auf. Was würden Sie hier empfehlen? Neben der Verwendung von Netzen, bedeckender Kleidung und Repellents fragen die Patienten auch nach der Wirksamkeit von MückenSpiralen. Sind diese zusätzlich zu empfehlen oder überflüssig?

Prof. Dr. Tino F. Schwarz, Würzburg: Generell sollte in Gebieten mit vektorübertragenen Infektionen auf eine konsequente Expositionsprophylaxe geachtet werden. Hierzu sind alle Permethrinoder DEET-haltigen Präparate geeignet. Welches Präparat genommen wird, ist eine individuelle Entscheidung (Reizung, Geruch, etc.). Bei Kindern sollten nur Präparate genommen werden, die dafür gekennzeichnet sind. Nicht-imprägnierte Moskitonetze sind für Kinder ebenfalls besser geeignet. Bei Mückenspriralen ist zu bedenken, dass diese Präparate Neurotoxine freisetzen. Es gibt kein 100\% sicheres Präparat - der Urlauber muss selbst herausfinden, was für ihn am besten wirkt. 